\title{
Learning defects in Drosophila growth restricted chico mutants are caused by attenuated adenylyl cyclase activity
}

\author{
Shintaro Naganos, Kohei Ueno, Junjiro Horiuchi and Minoru Saitoe*
}

\begin{abstract}
Background: Reduced insulin/insulin-like growth factor signaling (IIS) is a major cause of symmetrical intrauterine growth retardation (IUGR), an impairment in cell proliferation during prenatal development that results in global growth defects and mental retardation. In Drosophila, chico encodes the only insulin receptor substrate. Similar to other animal models of IUGR, chico mutants have defects in global growth and associative learning. However, the physiological and molecular bases of learning defects caused by chico mutations, and by symmetrical IUGR, are not clear.

Results: In this study, we found that chico mutations impair memory-associated synaptic plasticity in the mushroom bodies (MBs), neural centers for olfactory learning. Mutations in chico reduce expression of the rutabaga-type adenylyl cyclase (rut), leading to decreased cAMP synthesis in the MBs. Expressing a rut ${ }^{+}$transgene in the MBs restores memoryassociated plasticity and olfactory associative learning in chico mutants, without affecting growth. Thus chico mutations disrupt olfactory learning, at least in part, by reducing CAMP signaling in the MBs.

Conclusions: Our results suggest that some cognitive defects associated with reduced IIS may occur, independently of developmental defects, from acute reductions in CAMP signaling.
\end{abstract}

Keywords: Insulin signaling, CAMP, Synaptic plasticity, Associative learning, Intrauterine growth retardation

\section{Background}

Intrauterine growth retardation (IUGR) causes growth and psychological defects in children, and can be categorized into symmetrical and asymmetrical types [1]. Symmetrical IUGR is caused by early intrauterine disruptions, which lead to global decreases in cell proliferation, reduced brain size and cognitive deficits [2]. Asymmetrical IUGR is caused by poor nutrition or oxygen supply to the fetus during the third trimester of pregnancy, leading to decreased body size, but normal or near normal brain sizes. Symmetrical IUGR phenotypes include reduced numbers of neurons in the hippocampus and the cerebellum in neonates [3], and these reductions are proposed to explain cognitive deficits observed in symmetric IUGR. However, the actual mechanisms causing cognitive defects upon symmetrical IUGR are still unknown.

* Correspondence: saito-mn@igakuken.or.jp

Tokyo Metropolitan Institute of Medical Science, 2-1-6, Kamikitazawa, Setagaya 185-8506Tokyo, Japan
Mutations in insulin/insulin-like growth factor signaling (IIS) are associated with symmetrical IUGR $[4,5]$. In Drosophila, homozygous mutations in the chico gene, which encodes the only insulin receptor substrate in flies, decrease cell proliferation, reduce global body size and cause frequent death at birth [6-8]. Although chico mutants also display impaired associative learning [9], the physiological and molecular bases of learning defects caused by chico mutations are not clear.

During Drosophila olfactory aversive learning, flies learn to associate an odor with aversive electric shocks [10]. Olfactory associations are formed in central brain areas called the mushroom bodies (MBs) [11]. Information about the odor or conditioned stimulus (CS), is transmitted to the MBs by cholinergic projection neurons (PNs) from the antennal lobes (ALs) [12-14], while information about the electric shocks or unconditioned stimuli (US), are proposed to be transmitted to the MBs by dopaminergic neurons, which receive somatosensory information from the ascending fibers of the ventral nerve cord (AFV) [15-19]. Previously, using functional imaging of dissected, 
cultured brains, we showed that simultaneous stimulation of $\mathrm{AL}$ and $\mathrm{AFV}$ inputs to the MBs induces long-term enhancement (LTE) of synaptic transmission from the ALs to the MBs. We further showed that this LTE is a likely cellular correlate of olfactory aversive memory [20].

In this study, we found that chico mutants are defective for LTE, and have decreased expression of the rutabaga (rut) gene. rut encodes an adenylyl cyclase (rut-AC) required for cAMP signaling during associative learning. rut mutants have impaired learning $[10,21]$, and we show that expression of a rut ${ }^{+}$transgene in the MBs restores both learning and LTE in chico mutants. rut ${ }^{+}$expression restores learning and LTE without increasing numbers of $\mathrm{MB}$ neurons, indicating that learning defects in chico mutants are caused by reduced cAMP signaling, and not by reduced cell number. This suggests that some of the cognitive defects caused by symmetrical IUGR may occur independently of reduced cell number.

\section{Results}

\section{LTE is suppressed in chico ${ }^{1}$ mutants}

Chico mutants are defective for olfactory learning [9]. To determine whether they are also defective for memoryassociated plasticity, we examined LTE in brains of chico mutants expressing the $\mathrm{Ca}^{2+}$ indicator G-CaMP 1.3 in the MBs (Fig. 1a). chico $^{1}$ mutations consist of a P-element insertion 80 bp downstream from the translational start site, resulting in a complete loss of protein $[6,9]$. LTE is typically observed at the distal ends of the vertical lobes $\left(\alpha / \alpha^{\prime}\right)$ of the MBs, and is induced upon simultaneous stimulation of the ALs and AFV (AL + AFV in Fig. 1b), but not upon AL or AFV stimulation alone [20].

$\mathrm{Ca}^{2+}$ responses in the MBs induced by $\mathrm{AL}$ or AFV stimulation alone were indistinguishable between chico $^{1}$ and control brains (Fig. 1c and d). However, the increase in AL-induced $\mathrm{Ca}^{2+}$ responses after simultaneous stimulation of the AL and AFV (AL + AFV), observed in the control brains (UAS-G-CaMP/+; OK107/+), was suppressed in chico brains (chico ${ }^{1}$; UAS-G-CaMP/+; OK107/+) (Fig. 1e and f). This indicates that basal synaptic transmission from the ALs and AFV to the MBs is unaffected in chico mutants, but LTE is disrupted. Olfactory learning is restored in chico $^{1}$ flies by expressing a chico ${ }^{+}$transgene in the MBs [9]. Consistent with this, expressing a chico $^{+}$transgene in the MBs (chico ${ }^{1}, U A S-$ chico; UAS-G-CaMP/+; OK107/+) also restored LTE in chico ${ }^{1}$ brains (Fig. 1e and f), demonstrating that defects in LTE are caused by reduced chico expression in the MBs.

\section{Chico mutations decrease expression of rutabaga and synthesis of CAMP in the MBs}

We previously demonstrated that AL-induced $\mathrm{Ca}^{2+}$ responses in the MBs are mediated by nicotinic acetylcholine receptors (nAChRs), while AFV-induced $\mathrm{Ca}^{2+}$ responses in the MBs are mediated by NMDA receptors (NRs). Besides nAChRs and NRs, a third type of receptor, the D1-type dopamine receptor (D1R), encoded by the dopr gene, is also required for LTE formation [20]. To investigate the molecular bases of LTE defects in chico mutants, we examined expression of genes associated with these receptors (Fig. 2a). As expected from the normal AL- and AFVinduced $\mathrm{Ca}^{2+}$ responses in chico brains (Fig. 1c and d), expression of NRs ( $n r 1$ and $n r 2$ ), and choline acetyltransferase (chat), which is required for synthesis of acetylcholine, was unaltered in chico mutants. Likewise, expression of dopr, a second D1R subtype encoded by dopamine receptor 2 (dopr 2 ), and the D2-type dopamine receptor encoded by $d 2$-like dopamine receptor $(d 2 r)$, were also unchanged in chico mutants.

Cholinergic, glutamatergic and dopaminergic inputs affect various downstream signaling pathways, including the cAMP pathway. In particular, an adenylyl cyclase (AC) encoded by the rutabaga (rut) gene and a phosphodiesterase (PDE) encoded by the dunce $(d n c)$ gene are preferentially expressed in the MBs, and play critical roles in olfactory learning and synaptic plasticity [10, 22, 23]. While we did not observe significant changes in expression of $d n c$, or of the G-protein Gs alpha subunit $(g \alpha s)$ or the Gi alpha subunit $(g \alpha i)$, we found a significant decrease in rut expression in chico mutants compared to controls. Furthermore, expression of a chico $^{+}$transgene in the MBs restored rut expression to control levels in chico flies. Drosophila have 12 other adenylyl cyclase genes besides rut [24], four of which show high expression in the brain [25]. However, we did not observe significant changes in expression of these other brain-expressed AC genes in chico mutants compared to wild-type (Fig. 2b), indicating that chico specifically regulates expression of rut-AC.

Decreases in rut expression suggest that chico mutants may be defective for cAMP synthesis in the MBs. To test this possibility, we expressed a fluorescence resonance energy transfer (FRET) based cAMP probe, Epac1-camps [26, 27], in the MBs (Fig. 3). Using this probe, intracellular cAMP levels can be monitored as increases in the CFP/YFP ratio (decreasing FRET signal). When we applied $100 \mu \mathrm{M}$ forskolin, an activator of $\mathrm{AC}$, in the presence of IBMX, a cyclic nucleotide phosphodiesterase inhibitor, we observed a robust change in FRET signals in the vertical lobes $(\alpha / \alpha$ ' lobes) of the MBs of control brains (UAS-Epac1-camps/+; OK107/+) (Fig. 3a and b). In chico mutant brains (chico ${ }^{1}$; UAS-Epac1-camps/+; OK107/+), this change was significantly attenuated after the same treatment (Fig. 3a and b). Furthermore, this attenuated signal was rescued by expressing a chico ${ }^{+}$transgene in the MBs (chico ${ }^{1}$,UAS-chico; UAS-Epac1-camps/+; OK107/+) (Fig. 3a-c). These results suggest that chico regulates cAMP production in the MBs. 


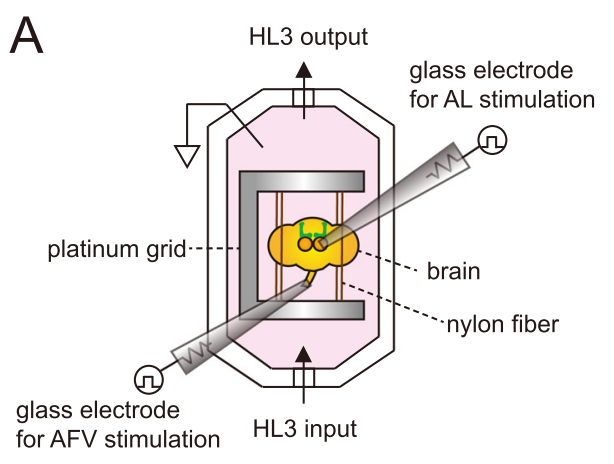

B

associative stimulation

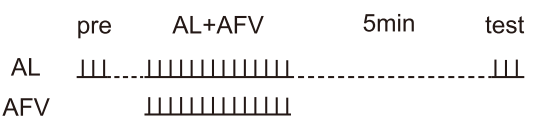

C

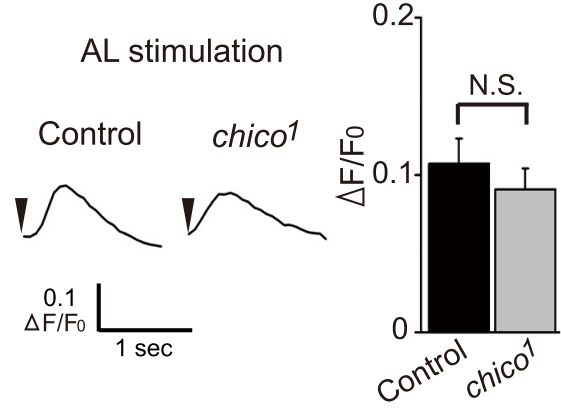

D

E
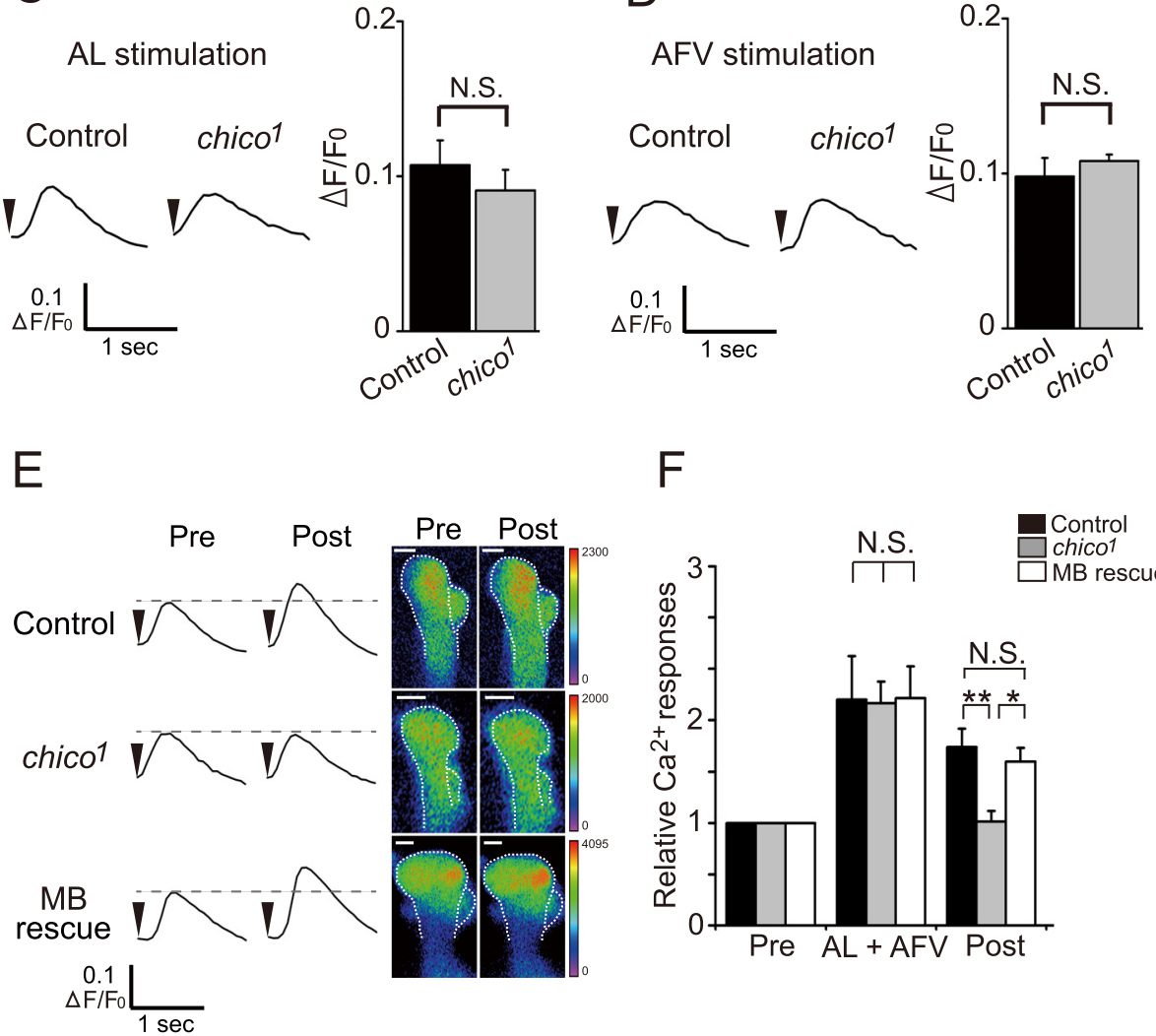

F
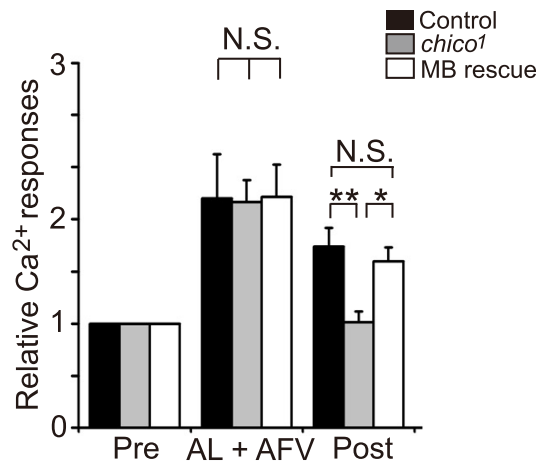

Fig. 1 Chico expression in the MBs is required for LTE of AL induced MB responses. a Schematic diagram of the recording setup. A dissected brain, held on a platinum grid by nylon fibers, is placed in a recording chamber perfused with HL3. Glass electrodes are used to electrically stimulate the AL and AFV, and resulting fluorescent signals are measured using a confocal microscope. See Materials and Methods for details. $\mathbf{b}$ Stimulation protocol. AL-induced Ca ${ }^{2}$ ${ }^{+}$responses before (pre) and after (test/post) simultaneous AL and AFV stimulation (AL + AFV) were used to quantify LTE. Vertical tick marks represent AL or AFV stimulation. $\mathbf{c}$ and $\mathbf{d}$ Typical traces of AL-induced (c) and AFV-induced (d) Ca ${ }^{2+}$ responses in control (UAS-G-CaMP/+; OK107/+) and chico (chico'; UAS-G-CaMP/+; OK107/+) brains, and summary graphs. $N=6$ for all data. e Typical AL-induced $\mathrm{Ca}^{2+}$ responses at the distal end of a/a' lobes of the MBs before (pre) and after (post) simultaneous AL and AFV stimulation. Scale bar, $10 \mu \mathrm{m}$. f Summary of $\mathrm{Ca}^{2+}$ responses obtained from control, chico ${ }^{1}$ and MB-chico rescue (chico',UAS-chico/chico'; UAS-G-CaMP/+; OK107/+) brains. Arrowheads indicate stimulation onset. $N=6$ for each data point. ** $P<0.01, * P<0.05$, N.S. Not Significant. Data are shown as means \pm SEM

\section{Expression of a rut ${ }^{+}$transgene in the MBs rescues learning defects of chico mutants}

We next tested whether decreased rut expression in the MBs could be responsible for impaired LTE, cAMP production and learning in chico mutants. When a rut ${ }^{+}$ transgene was expressed in the MBs, LTE in chico mutants was restored to wild-type levels (Fig. 4a and b), while $\mathrm{Ca}^{2+}$ responses evoked by AL- or AFV stimulation alone were not affected (Fig. 4c and d). Forskolin-induced Epac1-camps FRET signals in chico mutants were also partially restored by $\mathrm{MB}$ expression of the $r u t^{+}$transgene (Fig. 4e and f). Furthermore, MB expression of the rut ${ }^{+}$ 


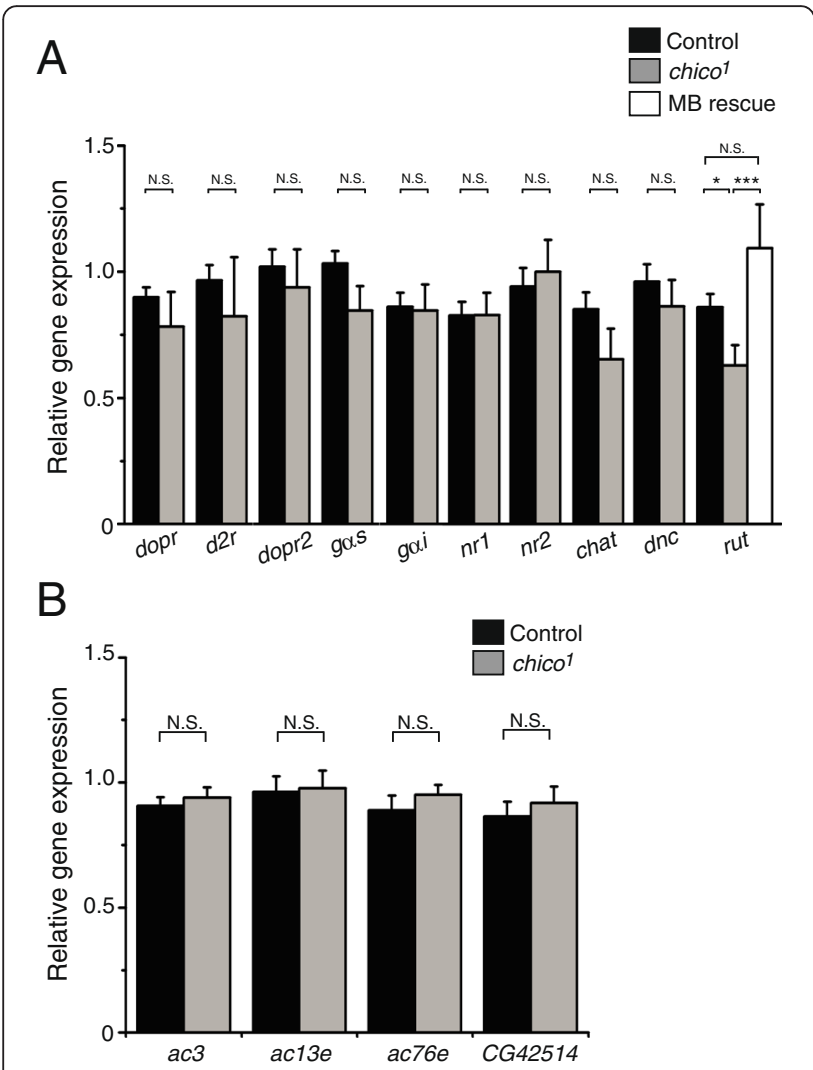

Fig. 2 Rutabaga expression is reduced in chico mutants. a Quantitated mRNA amounts in control (black), chico ${ }^{7}$ homozygote (gray) and MBchico rescued (chico', UAS-chico ${ }^{+} /$chico $^{1}$; OK107/+) fly head extracts. $N>5$ for each data point. $\mathbf{b}$ Expression of other putative adenylyl cyclases, encoded by ac3, ac13e, ac76e and CG42514, is not altered in chico ${ }^{\prime}$ mutants. $N=8$. All data in this figure are normalized to total RNA, and are shown as means \pm SEM. ${ }^{* *} P<0.001,{ }^{*} P<0.05$, N.S. Not Significant

transgene rescued learning (measured $3 \mathrm{~min}$ after conditioning) in chico flies (Fig. 5a). Taken together, these results indicate that decreased rut expression in the MBs is the likely cause of the reduced cAMP production, and impaired LTE and learning observed in chico mutants.

A previous study has reported that overexpressing AC can enhance memory retention in wild-type mice [28], raising an alternate possibility that $\mathrm{MB}$ expression of the $\mathrm{rut}^{+}$transgene may enhance olfactory learning in general, through a mechanism unrelated to chico rescue. Although we did not observe an increase in learning upon MB overexpression of the $\mathrm{rut}^{+}$transgene in a wild-type background (Fig. 5b, right panel), normal 1 min-duration olfactory conditioning induces a maximal, plateau-level amount of learning in wild-type flies, where increases may not be observable $[9,29,30]$. Thus, we employed short, 5 sec-duration conditioning trials, which produce lower learning indices, to assess learning enhancement upon rut $^{+}$overexpression in an otherwise wild-type background. As seen in the left panel of Fig. 5b, learning after $5 \mathrm{sec}$ conditioning does not increase in flies overexpressing $r^{+} t^{+}$in the MBs (UAS-rut/+;OK107/+). These results indicate that MB expression of $r u t^{+}$does not enhance learning in general, but specifically rescues learning defects in chico flies.

To address whether decreased rut expression in the MBs specifically at the adult stage causes learning defects in chico flies, we acutely expressed the $\mathrm{rut}^{+}$transgene from a MB-Geneswitch driver [31]. When chico flies were fed RU for 4 days prior to training, we observed partial but significant restoration of learning (Fig. 5c), suggesting that decreased rut activity in adult chico MBs contributes to learning defects in chico flies.

\section{MB expression of rut ${ }^{+}$does not restore MB growth in chico mutants}

Both learning defects and reduced Kenyon cell (MB intrinsic cell) number in chico mutants are rescued by expressing a chico ${ }^{+}$transgene in the MBs [9]. To determine whether the rescue of chico LTE and learning defects by $\mathrm{MB} \mathrm{rut} t^{+}$expression is also associated with increases in Kenyon cell number, we counted numbers of Kenyon cells in wild-type, chico $^{1}$ and chico $^{1} \mathrm{MB} \mathrm{rut}{ }^{+}$rescue brains. As seen in Fig. 6, chico $^{1}$ mutants have reduced numbers of Kenyon cells and $\mathrm{MB} \mathrm{rut} t^{+}$expression does not rescue this phenotype. Thus, cAMP signaling and learning defects in chico mutants occur independently of defects in cell proliferation, and at least some of the learning defects observed in chico mutants can be rescued in the absence of increasing cell number.

\section{Discussion}

Drosophila chico mutations and symmetrical IUGR are associated with several similar phenotypes, including decreased cell proliferation, global growth restriction, significant lethality at birth and learning defects $[6,9]$. In this study, we investigated the physiological and molecular bases of learning defects caused by chico mutations, and found that mutants are defective for synaptic plasticity, measured as LTE of AL-induced MB responses. chico mutants have normal olfactory avoidance and shock reactivity [9], and normal basal AL-induced and AFV-induced $\mathrm{Ca}^{2+}$ responses in the MBs, suggesting that they are defective for forming associations rather than transmitting sensory information. Expression of a chico ${ }^{+}$transgene in the MBs restores both olfactory learning and LTE in chico mutants. Thus, we propose that impaired LTE is likely to be physiologically correlated with learning defects in chico mutants.

We further found that chico mutants express significantly reduced amounts of rut. In comparison, expression of various other genes, including those involved in dopaminergic, glutamatergic and cholinergic signaling, is normal. Expressing a chico ${ }^{+}$transgene in the MBs restores normal rut expression, and normal cAMP levels in chico 

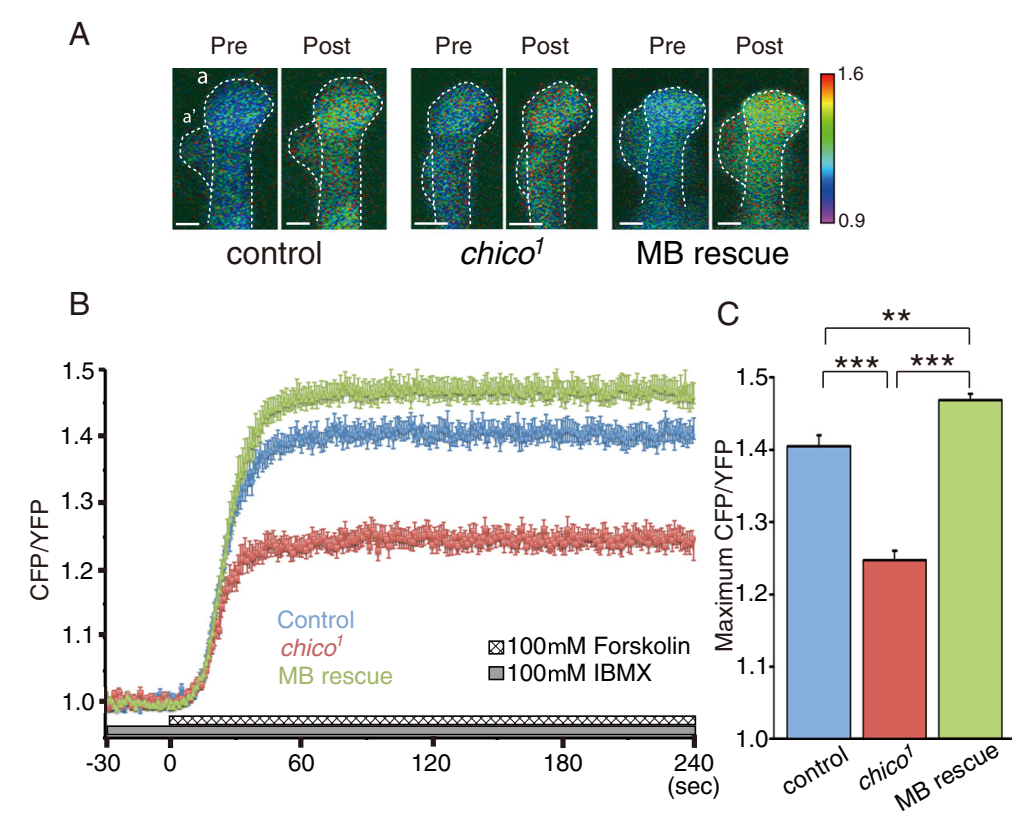

Fig. 3 Adenylyl cyclase-dependent CAMP synthesis is reduced in chico ${ }^{7}$ mutants. a Typical FRET images observed at the distal end of the a/a' MB lobe $10 \mathrm{sec}$ before (pre), and $60 \mathrm{sec}$ after (post), forskolin stimulation in brains from control (UAS-Epac1-camps/+; OK107/+), chico' (chico' ${ }^{\prime}$; UAS-Epac1camps/+; OK107/+) and MB-chico rescue (chico', UAS-chico/chico'; UAS-Epac1-camps/+; OK107/+) flies. A shift towards red indicates an increased CFP/ YFP ratio and increased CAMP amounts. Scale bar, $10 \mu \mathrm{m}$. b Time course of FRET changes (CFP/YFP) during forskolin stimulation. c Summary of maximum FRET changes (maximum CFP/YFP) upon forskolin stimulation. $N=14$ for control lines, $N=13$ for chico' mutants and MB rescue lines. ${ }^{* *} P<0.001$, and ${ }^{* *} P<0.01$. Data are shown as means \pm SEM

MBs, suggesting that chico likely regulates rut in a cell autonomous manner.

Rut-AC and its mammalian orthologue, type I AC, play critical roles in memory-associated plasticity and memory retention [10, 32, 33]. In flies, rut expression, specifically in the MBs, is required for associative learning [33, 34]. Consistent with this, we found that restoring Rut-AC function in the MBs is sufficient to partially restore forskolin-induced cAMP synthesis, and completely restore LTE and olfactory learning in chico mutants.

MB rut expression, specifically in adults, is required for adult olfactory learning [31,35], and we also found that acute $r u t^{+}$expression in adults significantly improves learning in chico mutants. Previously, it has been reported that increasing cAMP amounts increases synaptic transmission at the Drosophila larval neuromuscular junction [23]. However, expressing rut ${ }^{+}$in the MBs did not affect basal AL-induced and AFV-induced $\mathrm{Ca}^{2+}$ responses in the MBs, suggesting that Rut-AC, which is activated by both G-protein and $\mathrm{Ca}^{2+} /$ calmodulin signaling [32], functions during detection of coincident sensory stimuli, rather than during simple synaptic transmission.

Although increasing type I AC activity enhances memory in mice [28], we found that expressing a $\mathrm{rut}^{+}$transgene in the MBs does not enhance 3-min memory in flies from a wild-type background. Thus, AC seems to be a limiting factor in learning and memory formation in mice, while $\mathrm{MB}$ rut-AC amounts do not seem to be limiting in flies. Consistent with this idea, we found that expressing a rut $^{+}$transgene in the MBs at levels that only partially restore cAMP amounts, is enough to fully rescue LTE and learning defects in chico mutants. We propose that the $27 \%$ reduction in rut-AC expression observed in chico mutants (Fig. 2) reduces AC protein amounts below a threshold, such that insufficient amounts of cAMP are produced after coincident stimulation. This leads to deficiencies in both LTE and learning. Mild transgenic expression of rut ${ }^{+}$in the MBs may not restore rut expression to wild-type levels, but does increase expression enough to restore normal LTE and learning.

It is unclear why transgenic expression of rut ${ }^{+}$was insufficient to completely restore cAMP amounts to wild-type levels in chico mutants, since we expressed rut ${ }^{+}$from a strong OK107 driver. Since the chico mutation affects endogenous rut expression, it is possible that chico also affects expression of other genes including expression from the $\mathrm{OK} 107$ promoter. In addition, insulin signaling affects the target of rapamycin (TOR) kinase, which regulates protein translation [36, 37]. Thus chico may affect both transcription and translation, such that $\mathrm{rut}^{+}$transgene expression only partially rescues chico defects.

Although cAMP signaling is known to regulate IIS $[38,39]$, there have only been a few previous studies of 

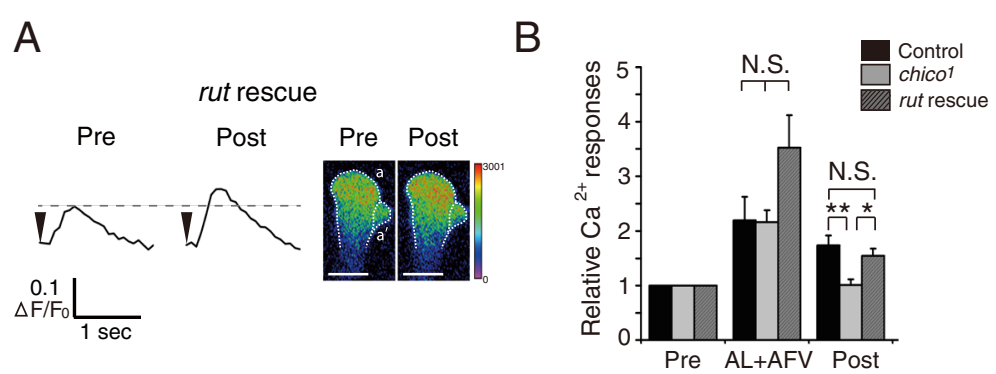

C

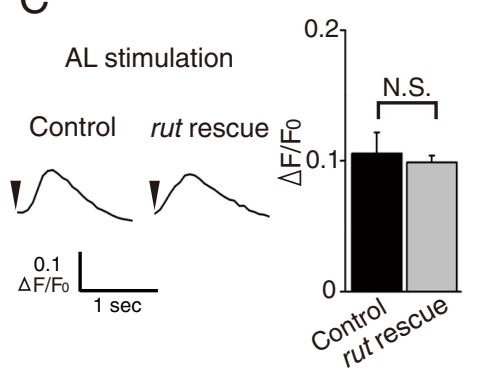

D
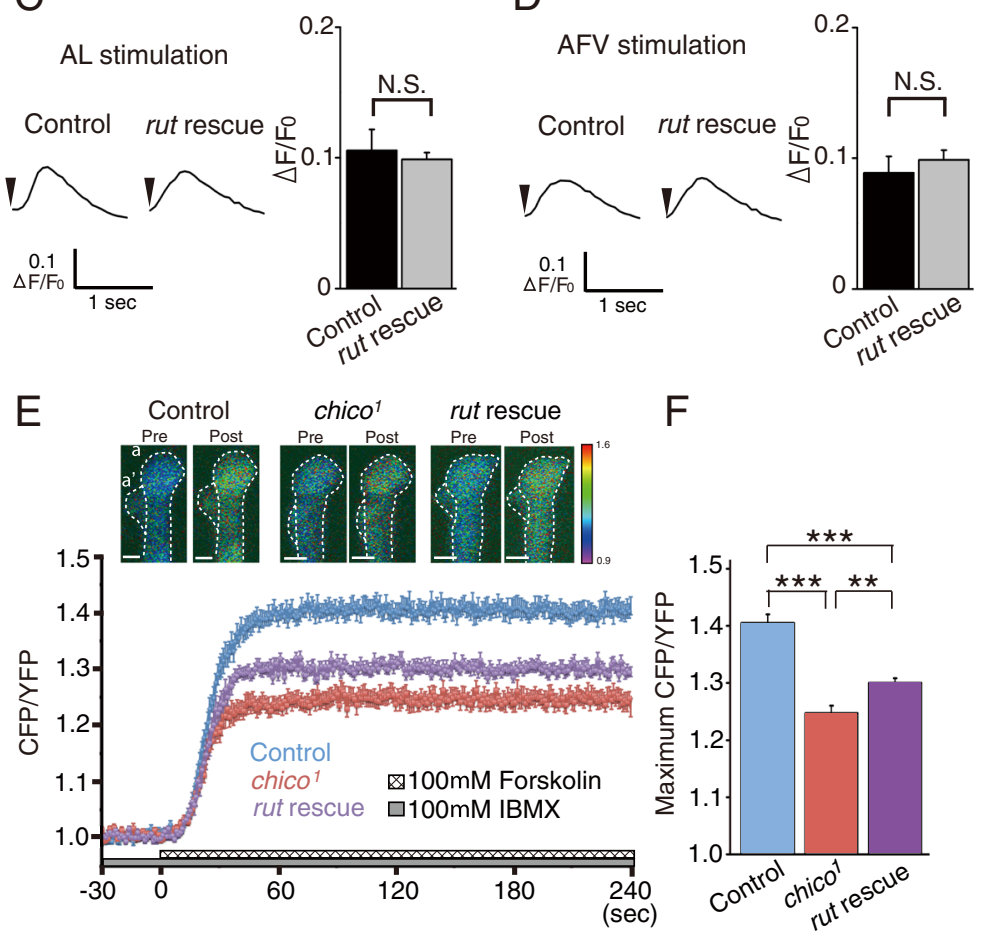

$\mathrm{F}$

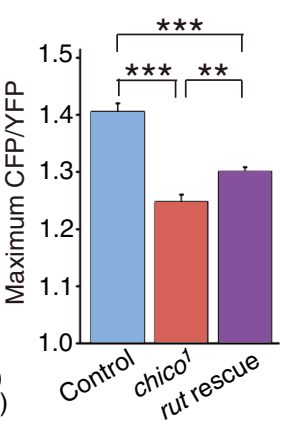

Fig. $4 \mathrm{MB}$ expression of a rut ${ }^{+}$transgene is sufficient to restore LTE formation and increase CAMP synthesis in chico mutants. $\mathbf{a}, \mathbf{b} \mathrm{MB}$ expression of the rut ${ }^{+}$transgene restores LTE in chico mutants. a Typical AL-induced $\mathrm{Ca}^{2+}$ responses before (pre) and 5 min after (post) AL + AFV stimulation in chico', UAS-rut/chico'; UAS-G-CaMP/+; OK107/+ (rut rescue) brains. Scale bar, 5 mm. b Summary of $\mathrm{Ca}^{2+}$ responses in control, chico and rut rescued flies. $\mathbf{c}$, d Typical traces (left) and average maximal responses (right) of AL-induced (c) and AFV-induced (d) $\mathrm{Ca}^{2+}$ responses in the $\mathrm{MBs}$ of $\mathrm{chico}^{1}$ (control) and rut rescue brains. $N=6$ for all data in $\mathbf{a}-\mathbf{d}$. e, $\mathbf{f} \mathrm{MB}$ expression of a rut ${ }^{+}$transgene increases CAMP synthesis in chico ${ }^{1}$ mutants upon forskolin stimulation. e Typical FRET images and time course (normalized CFP/YFP) upon forskolin stimulation in control (UAS-Epac1-camps/+; OK107/+, N=7), chico ${ }^{1}$ (chico'; UAS-Epac1-camps/+; OK107/t, N = 8) and rut rescue (chico', UAS-rut/chico'; UAS-Epac1-camps/+; OK107/t, N=6) brains. Scale bar, $10 \mu \mathrm{m}$. f Summary of maximum CFP/YFP ratios upon forskolin stimulation. N.S. Not Significant, ${ }^{* *} P<0.001,{ }^{* *} P<0.01$. Data are shown as means \pm SEM

IIS affecting cAMP. Of these, all have focused on IIS regulation of cAMP degradation by phosphodiesterases $[40,41]$. Ours is the first to report that IIS regulates cAMP production by controlling adenylyl cyclase expression. IIS activates the AKT kinase, which then phosphorylates and inactivates the forkhead transcription factor, Foxo $[36,42]$. Thus it is possible that reduced IIS activates Foxo, which in turn induces expression of a transcriptional repressor of rut. It will be of great interest in the future to determine whether IIS regulates rut expression through Foxo dependent or independent mechanisms.

\section{Conclusions}

We found that chico mutations impair learning and synaptic plasticity due to reduced expression and activity of the Rut-AC in the MBs. Attenuated AC activity is also associated with human IUGR, and stimulation-dependent cAMP production is defective in cultured fibroblasts derived from human patients with pre- and postnatal growth retardation, mental retardation and insulin resistance [43]. Given these similarities, our results may provide novel insights into the causes of mental retardation associated with symmetrical IUGR. 

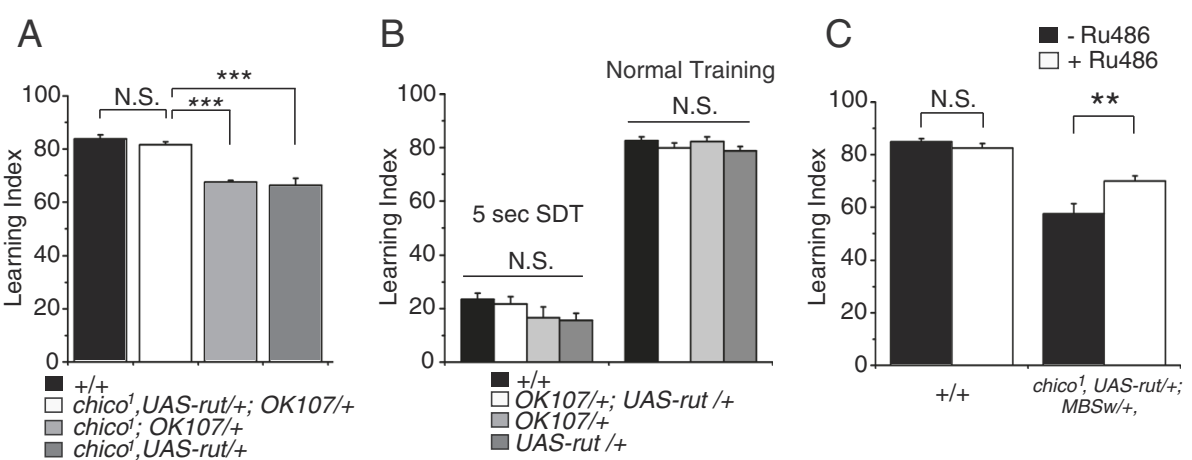

Fig. $5 \mathrm{MB}$ expression of a rut ${ }^{+}$transgene ameliorates learning defects of chico mutants. a MB expression of a rut ${ }^{+}$transgene rescues learning (3 min memory) in chico mutants. $N=8$. $\mathbf{b}$ Learning (3 min memory) is unaffected by MB overexpression of a rut ${ }^{+}$transgene in a wild type background. No significant differences in performance were observed after either $5 \mathrm{sec}$ short duration training (SDT) or 60 sec normal training. $N=7-14$. $\mathbf{c}$ Learning is improved in chico mutants by expressing a rut ${ }^{+}$transgene from a MB-Geneswitch driver. $N=8$. N.S. Not Significant, ${ }^{* * *} P<0.001,{ }^{* *} P<0.01$. All Data in this figure are shown as means \pm SEM

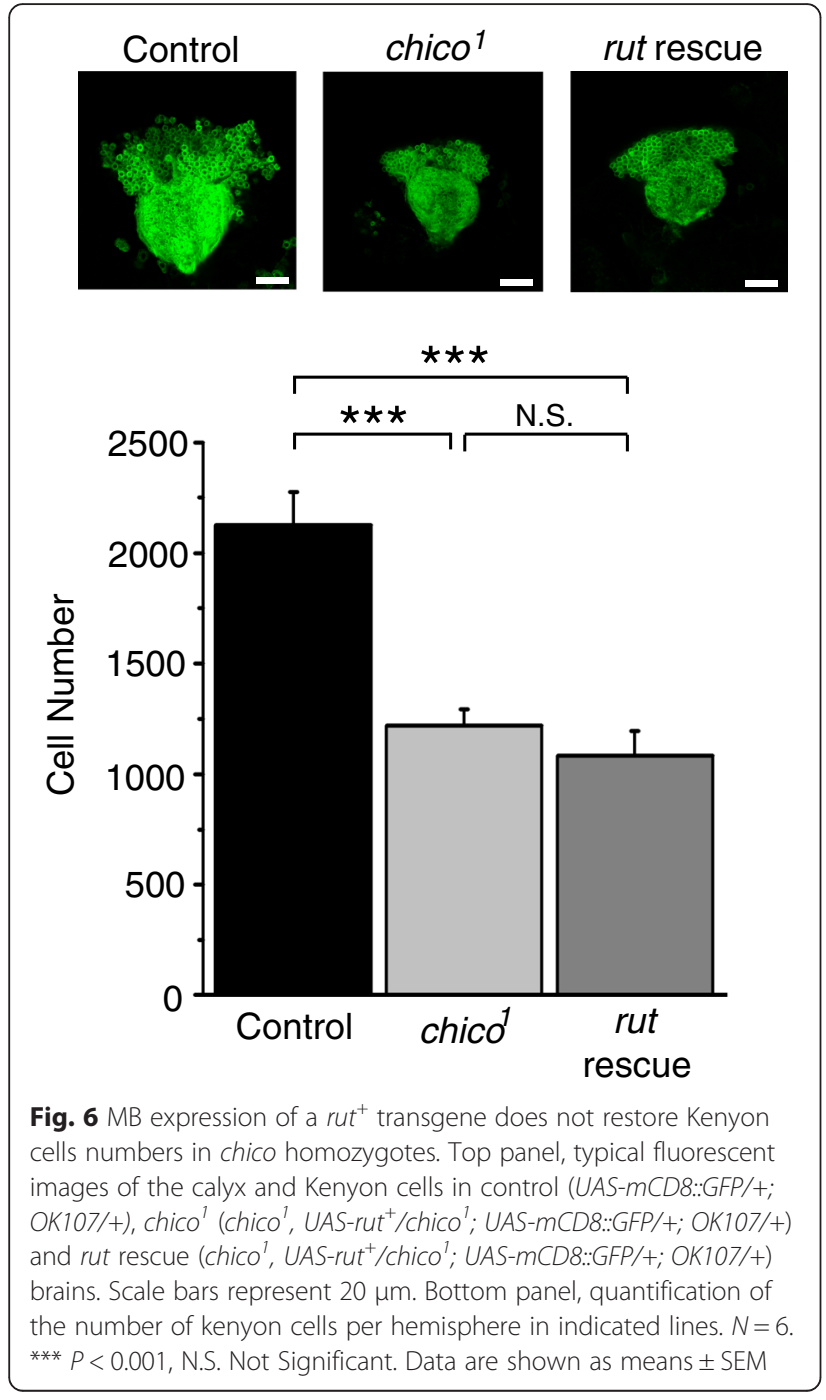

\section{Methods}

Fly stocks

All fly stocks were maintained at $25 \pm 2{ }^{\circ} \mathrm{C}$ and $60 \pm$ $10 \%$ humidity under a $12 \mathrm{~h} / 12 \mathrm{~h}$ light dark cycle. All transgenic flies and mutants were outcrossed to our wild-type control line $w(C S 10)$ [44] between 5 and 10 times. For imaging analyses, we used female flies, since chico homozygous flies are semi-lethal and homozygous males rarely emerged in our genetic background. For behavioral tests, we used both male and female flies.

\section{Imaging analysis Imaging preparation}

Brains with attached ventral nerve cords (VNC) were dissected in $0 \mathrm{mM} \mathrm{Ca}{ }^{2+} \mathrm{HL} 3$ medium $[\mathrm{NaCl}, 70 \mathrm{mM}$; sucrose, $115 \mathrm{mM}$; KCl, $5 \mathrm{mM} ; \mathrm{MgCl}_{2}, 20 \mathrm{mM} ; \mathrm{NaHCO}_{3}$, $10 \mathrm{mM}$; trehalose, $5 \mathrm{mM}$; and HEPES, $5 \mathrm{mM}$, pH 7.3] [45]. The ventral nerve cord was then cut at the cervical connective and isolated brains were immobilized by placing their optic lobes between two nylon fiber bundles stretched across a platinum grid, and placed in a bath chamber (Fig. 1a). ALs and AFV were electrically stimulated using glass micro-electrodes and stimulators (SEN7103, Nihon Kohden, Japan) and isolators (SS-104 J for AL, SS202J for AFV, Nihon Kohden). During experiments, fresh HL3 [ $\mathrm{NaCl}, 70 \mathrm{mM}$; sucrose, $115 \mathrm{mM} ; \mathrm{KCl}$, $5 \mathrm{mM} ; \mathrm{MgCl}_{2}, 20 \mathrm{mM} ; \mathrm{CaCl}_{2}, 1.5 \mathrm{mM} ; \mathrm{NaHCO}_{3}$, $10 \mathrm{mM}$; trehalose, $5 \mathrm{mM}$; and HEPES, $5 \mathrm{mM}$, pH 7.3] was infused into the chamber using a peristaltic pump ( $3 \mathrm{ml} / \mathrm{min}$ Gilson, Inc.).

\section{$\mathrm{Ca}^{2+}$ imaging}

Images were captured using a confocal microscope system (A1, Nikon Corp., Japan). The objective lens was a $20 \times$ water-immersion lens (NA: 0.5 Nikon Corp.). Before experiments, we set the offset value so that the background 
intensity would be close to 0 . The $F$ value was calculated for each pixel in the region of interest (ROI) using NISelements software (NIS-Elements Ar, Nikon Corp.).

We obtained initial fluorescence values $\left(\mathrm{F}_{0}\right)$ by averaging values in the five sequential frames before stimulation onset. To record $\mathrm{AL}$ - and AFV-induced $\mathrm{Ca}^{2+}$ responses, we stimulated the AL or AFV with 3 trains of 30 pulses $(100 \mathrm{~Hz}, 1.0 \mathrm{~ms}$ pulse duration, at a current that produced a $10 \%$ increase in fluorescence compared to $\mathrm{F}_{0}$ ) with an inter-train interval of $10 \mathrm{sec}$. We then averaged three fluorescent traces after stimulation and used peak $F$ values to calculate $\Delta \mathrm{F} / \mathrm{F}_{0}$. To quantify LTE, we divided $\Delta \mathrm{F} / \mathrm{F}_{0}$ values obtained after associative stimulation of the AL and AFV by values obtained prior to associative stimulation.

\section{Electrical stimulation}

For coincident or "associative" stimulation, the AL and AFV were simultaneously stimulated with 12 trains (see above) with an inter-train interval of $5 \mathrm{sec}$, and the average peak value of the first three responses was used as the relative $\mathrm{Ca}^{2+}$ response during stimulation.

\section{CAMP imaging}

For cAMP imaging, dissected brains were incubated in HL3 medium containing $100 \mu \mathrm{M}$ IBMX for $10 \mathrm{~min}$ at room temperature. IBMX and forskolin were prepared as 250 and $100 \mathrm{mM}$ solutions in DMSO, and diluted in HL3 to $100 \mu \mathrm{M}$. CFP was excited at $457 \mathrm{~nm}$ and detected using a $482 \pm 17.5 \mathrm{~nm}$ band pass filter, and YFP was detected simultaneously using a $540 \pm 15 \mathrm{~nm}$ band pass filter. FRET changes were measured as changes in the ratio of CFP to YFP (CFP/YFP) signals. Signal changes were calculated for each pixel in the region of interest (ROI) using NISelements software. Images were recorded at 1 frame per second for $270 \mathrm{sec}$. Averaged CFP/YFP values in the twenty sequential frames before forskolin application were used for data normalization. Maximum CFP/YFP values were calculated by averaging CGP/YFP values in the last 120 frames of each experiment.

\section{Behavioral tests}

The procedure for measuring olfactory memory is described elsewhere [10]. Briefly, $\sim 100$ flies were exposed sequentially to two aversive odors, 3-octanol [OCT] and 4-methylcyclohexanol $[\mathrm{MCH}]$, for $1 \mathrm{~min}$ with an interval of $45 \mathrm{sec}$ between each odor exposure. When the flies were exposed by the first, $\mathrm{CS}+$ odor (either OCT or $\mathrm{MCH})$, they were also subjected to $1.5 \mathrm{sec}$ pulses of $60 \mathrm{~V} \mathrm{DC}$ electric shocks every $5 \mathrm{sec}$ (US). For shortduration training, flies were exposed to the CS+ and CSodors for $5 \mathrm{sec}$ each, and one shock pulse was given during CS+ exposure [9]. To test olfactory memory, flies were placed at the choice point of a T-maze where both $\mathrm{CS}+$ and CS- odors were delivered and allowed to choose between the odors. After $1.5 \mathrm{~min}$, flies choosing each odor were counted, and memory was calculated as a learning index, such that a 50:50 distribution (no memory) yielded a learning index of zero and a 0:100 distribution away from the CS+ yielded a learning index of 100 . For conditional expression from geneswitch drivers, flies were fed $1 \mathrm{mM}$ RU486 for 4 days prior to behavior experiments.

\section{Quantitative RT-PCR}

Total RNA was isolated from fly heads using TRIZOL reagent and reverse transcribed into cDNA using ReverTra Ace (TOYOBO) and random primers (TOYOBO). Quantitative PCR was performed using SYBR Green Realtime PCR Master Mix (TOYOBO) to measure gene expression. Primer sequences were,

dopr forward: 5' - CATGGGCGTTTTTCTCATCT -3'; dopr reverse: 5' - AGCCAGGTGAGGATCTTGAA -3'; $d 2 r$ forward: $5^{\prime}$ - GCGTGTTCATCATCTGTTGG -3'; $d 2 r$ reverse: $5^{\prime}$ - TCATGCAGGAGTTGATCCAG -3'; dopr 2 forward: 5' - GCGTGTTCATCATCTGTTGG -3'; dopr 2 reverse: 5' - TCATGCAGGAGTTGATCCAG -3'; rut forward: 5' - CGGGCTCATCCGTAATGTAG -3'; rut reverse: 5' - CCCATCTCAATCACCAGTCC -3'; $d n c$ forward: 5' - CGGAGAGTCGTGGGAATTTA -3'; $d n c$ reverse: $5^{\prime}$ - TTGCTTTTCGTTTTAGGTTTTTG -3'; gos forward: 5' - ACTGGAGCAGAACGGAGAAA - 3'; gas reverse: 5' - GAGTCCTCCGAGTTCACGTC -3'; gai forward: 5' - CGGTGGGTTTTGTACATCCT -3'; gai reverse:5' - GGATTTCTGGATGTGGTGCT -3'; $n r 1$ forward: 5' -TGTTGCAAGCCGTATACCAA-3'; nr1 reverse: 5 '-ACAGGCTATCGGAGCTTTCA-3'; $n r 2$ forward: 5' - GACTATCTGGTTGCCCAGGA-3'; nr2 reverse: 5' - ACTTTGAGTTGCGGCTGAAT-3'; chat forward: 5' -ATCATCTCGCAGTGCTTCCT-3'; chat reverse: 5' - AAGACGATCTGTTCCGGATG-3'; ac3 forward: 5' - AAATGACGCCCCAATTACAG-3'; ac3 reverse: 5' - GTCCATGATTGATCCCCATC-3'; ac13e forward: 5' - TTGCAGTGTCCCAGTTGATG-3'; ac13e reverse: 5' - AGACCAACACCAGGATGAGC-3'; ac76e forward: 5' - CAGGATGAATGACGCCCTTTC GG-3';

ac76e reverse: $5^{\prime}$ - ATGGACACAACACATGCCAG CAGC-3';

CG42514 forward: 5' - AATGCCTGGTTCCTTCAG TG-3';

CG42514 reverse: $5^{\prime}$ - TTCCCACTAGTCCCATGA GC-3';

\section{Whole mount immunohistochemistry and cell counting}

MB Kenyon cells were visualized by expressing $m C D 8:: G F P$ from the OK107 GAL4 driver in wild-type and chico backgrounds. Brains were dissected, fixed in PBS containing $4 \%$ PFA, and blocked in PBT containing $4 \%$ BLOCK 
ACE (DS Pharma medical). Brains were then incubated with primary antibodies (a 1:250 dilution of chick antiGFP polyclonal antibody (Abcam)) overnight at $4{ }^{\circ} \mathrm{C}$. Alexa Fluor488-conjugated goat anti-chick IgG (1:400; Invitrogen) was used for the secondary antibody. Images were captured using a FV500 microscope (Olympus Corp., Japan) with a 60x objective lens. We captured confocal image stacks of all MB Kenyon cells in each brain hemisphere with a $0.1 \mu \mathrm{m}$ interval between optical slices. Stacks of each brain were then analyzed manually to determine average cell diameters in the $\mathrm{z}$-axis. This tended to be about $0.275 \mu \mathrm{m}$ for wild-type Kenyon cells, and $0.2 \mu \mathrm{m}$ for chico mutants. Optical slices at these intervals were then manually counted for GFP positive cells to quantify the total number of Kenyon cells/hemisphere.

\section{Statistics}

We used unpaired two-tailed student's $t$-tests to evaluate statistical significance between two data sets and employed one-way ANOVA for multiple comparisons. Tukey's posthoc tests were used to determine significant individual differences after one-way ANOVA. All statistics were calculated using GraphPad Prism 5.02 (GraphPad Software).

\begin{abstract}
Abbreviations
AC: adenylyl cyclase; AFV: ascending fibers of the ventral nerve cord; ALs: antennal lobes; D1R: the D1-type dopamine receptor; dnc: dunce; FRET: fluorescence resonance energy transfer; IIS: insulin/insulin-like growth factor signaling; IUGR: intrauterine growth retardation; LTE: long-term enhancement; MBs: mushroom bodies; MCH: 4-methylcyclohexanol; nAChRs: nicotinic acetylcholine receptors; NRs: NMDA receptors; OCT: 3octanol; PDE: phosphodiesterase; PNs: projection neurons; ROI: region of interest; rut: rutabaga; TOR: target of rapamycin kinase; US: unconditioned stimuli; VNC: ventral nerve cords.
\end{abstract}

\section{Competing interests}

The authors declare that they have no competing interests.

\section{Authors' contributions}

SN designed and performed most of the experiments. KU contributed to the imaging study. MS supervised and wrote the manuscript with SN and JH. All authors read and approved the final manuscript.

\section{Acknowledgements}

We thank Yuki Kawabata for technical assistance. This work was supported by Grants-in-Aid for Scientific Research in Innovative Areas, "Memory dynamism" (25115006) and Scientific Research (A) (25250010) from the Ministry of Education, Culture, Sports, Science and Technology (MEXT) and grants from the Takeda Science Foundation, and The Mitsubishi Foundation to MS and a Grant for Basic Science Research Projects from The Sumitomo Foundation and Grant-in-Aid for Young Scientists (B) (15 K18577) from MEXT to SN.

Received: 14 January 2016 Accepted: 29 March 2016

Published online: 06 April 2016

\section{References}

1. Pollack RN, Divon MY. Intrauterine growth retardation: definition, classification, and etiology. Clin Obstet Gynecol. 1992;35:99-107.

2. Low JA, Handley-Derry MH, Burke SO, Peters RD, Pater EA, Killen HL, Derrick EJ. Association of intrauterine fetal growth retardation and learning deficits at age 9 to 11 years. Am J Obstet Gynecol. 1992;167:1499-505.

3. Mallard C, Loeliger M, Copolov D, Rees S. Reduced number of neurons in the hippocampus and the cerebellum in the postnatal guinea-pig following intrauterine growth-restriction. Neuroscience. 2000;100:327-33.
4. Powell-Braxton L, Hollingshead P, Warburton C, Dowd M, Pitts-Meek S, Dalton D, Gillett N, Stewart TA. IGF-I is required for normal embryonic growth in mice. Genes Dev. 1993;7:2609-17.

5. Woods KA, Camacho-Hubner C, Savage MO, Clark AJ. Intrauterine growth retardation and postnatal growth failure associated with deletion of the insulin-like growth factor I gene. N Engl J Med. 1996;335:1363-7.

6. Bohni R, Riesgo-Escovar J, Oldham S, Brogiolo W, Stocker H, Andruss BF, Beckingham K, Hafen E. Autonomous control of cell and organ size by CHICO, a Drosophila homolog of vertebrate IRS1-4. Cell. 1999;97:865-75.

7. Poltilove RM, Jacobs AR, Haft CR, Xu P, Taylor SI. Characterization of Drosophila insulin receptor substrate. J Biol Chem. 2000;275:23346-54.

8. Clancy DJ, Gems D, Harshman LG, Oldham S, Stocker H, Hafen E, Leevers SJ, Partridge L. Extension of life-span by loss of $\mathrm{CHICO}$, a Drosophila insulin receptor substrate protein. Science. 2001;292:104-6.

9. Naganos S, Horiuchi J, Saitoe M. Mutations in the Drosophila insulin receptor substrate, $\mathrm{CHICO}$, impair olfactory associative learning. Neurosci Res. 2012;73:49-55.

10. Tully T, Quinn WG. Classical conditioning and retention in normal and mutant Drosophila melanogaster. J Comp Physiol A. 1985;157:263-77.

11. Heisenberg M. Mushroom body memoir: from maps to models. Nat Rev Neurosci. 2003;4:266-75.

12. Schurmann FW. Acetylcholine, GABA, glutamate and NO as putative transmitters indicated by immunocytochemistry in the olfactory mushroom body system of the insect brain. Acta Biol Hung. 2000;51:355-62.

13. Yasuyama K, Meinertzhagen IA, Schurmann FW. Synaptic organization of the mushroom body calyx in Drosophila melanogaster. J Comp Neurol. 2002; 445:211-26.

14. Gu H, O'Dowd DK. Cholinergic synaptic transmission in adult Drosophila Kenyon cells in situ. J Neurosci. 2006;26:265-72.

15. Qin H, Cressy M, Li W, Coravos JS, Izzi SA, Dubnau J. Gamma neurons mediate dopaminergic input during aversive olfactory memory formation in Drosophila. Curr Biol. 2012;22:608-14.

16. Schwaerzel M, Monastirioti M, Scholz H, Friggi-Grelin F, Birman S, Heisenberg M. Dopamine and octopamine differentiate between aversive and appetitive olfactory memories in Drosophila. J Neurosci. 2003;23:10495-502.

17. Riemensperger T, Voller T, Stock P, Buchner E, Fiala A. Punishment prediction by dopaminergic neurons in Drosophila. Curr Biol. 2005;15:1953-60.

18. Aso Y, Herb A, Ogueta M, Siwanowicz I, Templier T, Friedrich AB, Ito K, Scholz $\mathrm{H}$, Tanimoto $\mathrm{H}$. Three dopamine pathways induce aversive odor memories with different stability. PLoS Genet. 2012;8:e1002768.

19. Aso Y, Siwanowicz I, Bracker L, Ito K, Kitamoto T, Tanimoto H. Specific dopaminergic neurons for the formation of labile aversive memory. Curr Biol. 2010;20:1445-51.

20. Ueno K, Naganos S, Hirano Y, Horiuchi J, Saitoe M. Long-term enhancement of synaptic transmission between antennal lobe and mushroom body in cultured Drosophila brain. J Physiol. 2013;591:287-302.

21. Han PL, Levin LR, Reed RR, Davis RL. Preferential expression of the Drosophila rutabaga gene in mushroom bodies, neural centers for learning in insects. Neuron. 1992;9:619-27.

22. Nighorn A, Healy MJ, Davis RL. The cyclic AMP phosphodiesterase encoded by the Drosophila dunce gene is concentrated in the mushroom body neuropil. Neuron. 1991;6:455-67.

23. Zhong Y, Wu CF. Altered synaptic plasticity in Drosophila memory mutants with a defective cyclic AMP cascade. Science. 1991;251:198-201.

24. Ueno K, Kidokoro Y. Adenylyl cyclase encoded by AC78C participates in sugar perception in Drosophila melanogaster. Eur J Neurosci. 2008;28:1956-66.

25. Chintapalli VR, Wang J, Dow JA. Using FlyAtlas to identify better Drosophila melanogaster models of human disease. Nat Genet. 2007;39:715-20.

26. Shafer OT, Kim DJ, Dunbar-Yaffe R, Nikolaev VO, Lohse MJ, Taghert PH. Widespread receptivity to neuropeptide PDF throughout the neuronal circadian clock network of Drosophila revealed by real-time cyclic AMP imaging. Neuron. 2008;58:223-37.

27. Nikolaev VO, Bunemann M, Hein L, Hannawacker A, Lohse MJ. Novel single chain CAMP sensors for receptor-induced signal propagation. J Biol Chem. 2004;279:37215-8.

28. Wang H, Ferguson GD, Pineda W, Cundiff PE, Storm DR. Overexpression of type-1 adenylyl cyclase in mouse forebrain enhances recognition memory and LTP. Nat Neurosci. 2004;7:635-42.

29. Cheng Y, Endo K, Wu K, Rodan AR, Heberlein U, Davis RL. Drosophila fasciclinll is required for the formation of odor memories and for normal sensitivity to alcohol. Cell. 2001;105:757-68. 
30. Tamura T, Chiang AS, Ito N, Liu HP, Horiuchi J, Tully T, Saitoe M. Aging specifically impairs amnesiac-dependent memory in Drosophila. Neuron. 2003;40:1003-11.

31. Mao Z, Roman G, Zong L, Davis RL. Pharmacogenetic rescue in time and space of the rutabaga memory impairment by using Gene-Switch. Proc Natl Acad Sci U S A. 2004;101:198-203.

32. Wu ZL, Thomas SA, Villacres EC, Xia Z, Simmons ML, Chavkin C, Palmiter RD, Storm DR. Altered behavior and long-term potentiation in type I adenylyl cyclase mutant mice. Proc Natl Acad Sci U S A. 1995;92:220-4.

33. Blum AL, Li W, Cressy M, Dubnau J. Short- and long-term memory in Drosophila require CAMP signaling in distinct neuron types. Curr Biol. 2009; 19:1341-50

34. Zars T, Fischer M, Schulz R, Heisenberg M. Localization of a short-term memory in Drosophila. Science. 2000;288:672-5.

35. McGuire SE, Le PT, Osborn AJ, Matsumoto K, Davis RL. Spatiotemporal rescue of memory dysfunction in Drosophila. Science. 2003;302:1765-8.

36. Teleman AA. Molecular mechanisms of metabolic regulation by insulin in Drosophila. Biochem J. 2010;425:13-26.

37. Holz MK, Ballif BA, Gygi SP, Blenis J. mTOR and S6K1 mediate assembly of the translation preinitiation complex through dynamic protein interchange and ordered phosphorylation events. Cell. 2005;123:569-80.

38. Shibasaki T, Takahashi H, Miki T, Sunaga Y, Matsumura K, Yamanaka M, Zhang C, Tamamoto A, Satoh T, Miyazaki J, Seino S. Essential role of Epac2/ Rap1 signaling in regulation of insulin granule dynamics by CAMP. Proc Natl Acad Sci U S A. 2007;104:19333-8.

39. Prentki M, Matschinsky FM. Ca2+, CAMP, and phospholipid-derived messengers in coupling mechanisms of insulin secretion. Physiol Rev. 1987;67:1185-248.

40. Kitamura T, Kitamura Y, Kuroda S, Hino Y, Ando M, Kotani K, Konishi H, Matsuzaki H, Kikkawa U, Ogawa W, Kasuga M. Insulin-induced phosphorylation and activation of cyclic nucleotide phosphodiesterase 3B by the serine-threonine kinase Akt. Mol Cell Biol. 1999;19:6286-96.

41. Wijkander J, Landstrom TR, Manganiello V, Belfrage P, Degerman E. Insulininduced phosphorylation and activation of phosphodiesterase $3 \mathrm{~B}$ in rat adipocytes: possible role for protein kinase B but not mitogen-activated protein kinase or p70 S6 kinase. Endocrinology. 1998;139:219-27.

42. Grewal SS. Insulin/TOR signaling in growth and homeostasis: a view from the fly world. Int J Biochem Cell Biol. 2009:41:1006-10.

43. Salerno M, Amabile G, Mandato C, Di Maio S, Lecora M, Avvedimento EV, Andria G. Growth retardation, developmental delay, distinctive face, multiple endocrine abnormalities, and adenylyl cyclase dysfunction: a new syndrome? Am J Med Genet A. 2003;120A:389-94.

44. Dura JM, Preat T, Tully T. Identification of linotte, a new gene affecting learning and memory in Drosophila melanogaster. J Neurogenet. 1993;9:1-14.

45. Stewart BA, Atwood HL, Renger JJ, Wang J, Wu CF. Improved stability of Drosophila larval neuromuscular preparations in haemolymph-like physiological solutions. J Comp Physiol A. 1994;175:179-91.

\section{Submit your next manuscript to BioMed Central and we will help you at every step:}

- We accept pre-submission inquiries

- Our selector tool helps you to find the most relevant journal

- We provide round the clock customer support

- Convenient online submission

- Thorough peer review

- Inclusion in PubMed and all major indexing services

- Maximum visibility for your research

Submit your manuscript at www.biomedcentral.com/submit

) Biomed Central 